\title{
Role of polymorphic Fc gamma receptor IIla and EGFR expression level in cetuximab mediated, NK cell dependent in vitro cytotoxicity of head and neck squamous cell carcinoma cells
}

\author{
Andrés López-Albaitero, \\ Department of Otolaryngology, University of Pittsburgh, Pittsburgh, PA, USA \\ Steve C. Lee, \\ Department of Otolaryngology, University of Pittsburgh, Pittsburgh, PA, USA \\ Sarah Morgan, \\ Department of Otolaryngology, University of Pittsburgh, Pittsburgh, PA, USA \\ Jennifer R. Grandis, \\ Department of Otolaryngology, University of Pittsburgh, Pittsburgh, PA, USA \\ Department of Pharmacology, University of Pittsburgh, Pittsburgh, PA, USA \\ William E. Gooding, \\ Biostatistics Facility, University of Pittsburgh Cancer Institute, Pittsburgh, PA, USA \\ Soldano Ferrone, and \\ Department of Surgery, University of Pittsburgh Cancer Institute, Pittsburgh, PA, USA \\ Department of Immunology, University of Pittsburgh Cancer Institute, Pittsburgh, PA, USA \\ Cancer Immunology, Immunotherapy and Immunoprevention Program, University of Pittsburgh \\ Cancer Institute, Pittsburgh, PA, USA

\section{Robert L. Ferris} \\ Department of Otolaryngology, University of Pittsburgh, Pittsburgh, PA, USA \\ Department of Immunology, University of Pittsburgh Cancer Institute, Pittsburgh, PA, USA \\ Cancer Immunology, Immunotherapy and Immunoprevention Program, University of Pittsburgh \\ Cancer Institute, Pittsburgh, PA, USA \\ The Hillman Cancer Center Research Pavilion, 5117 Centre Avenue, Room 2.26b, Pittsburgh, PA \\ 15213, USA
}

\section{Abstract \\ Immunotherapy with the EGFR-specific mAb cetuximab is clinically effective in 10-20\% of patients with squamous cell carcinoma of the head and neck (SCCHN). Little information is available about the mechanism(s) underlying patients' differential clinical response to cetuximab- based immunotherapy, although this information may contribute to optimizing the design of cetuximab-based immunotherapy. Our understanding of these mechanisms would benefit from the}

\footnotetext{
(C) Springer-Verlag 2009

Correspondence to: Robert L. Ferris.

ferrisrl@upmc.edu.
} 
characterization of the variables which influence the extent of cell dependent-lysis of SCCHN cells incubated with cetuximab in vitro. Therefore, in this study we have investigated the role of Fc $\gamma$ R IIIa-158 genotype expressed by effector NK cells, cetuximab concentration, and EGFR expression level by SCCHN cells in the extent of their in vitro lysis and in the degree of NK cell activation. PBMC or purified CD56 ${ }^{+}$NK cells genotyped at IIIa codon 158 and SCCHN cell lines expressing different levels of EGFR have been used as effectors and targets, respectively, in antibody dependent cellular cytotoxicity (ADCC) assays. Furthermore, supernatants from ADCC assays were analyzed for cytokine and chemokine levels using multiplexed ELISA. We found that the extent of lysis of SCCHN cells was influenced by the EGFR expression level, cetuximab concentration, and $\mathrm{Fc} \gamma \mathrm{R}$ polymorphism. Effector cells expressing the $\mathrm{Fc} \gamma \mathrm{R}$ IIIa-158 VV allele were significantly $(P<0.0001)$ more effective than those expressing $\mathrm{Fc} \gamma \mathrm{R}$ IIIa VF and VV alleles in mediating lysis of SCCHN cells expressed higher levels of the activation markers CD69 and CD107a, and secreted significantly $(P<0.05)$ larger amounts of inflammatory cytokines and chemokines. IL- 2 or IL- 15 treatment increased cetuximab-mediated ADCC by poor binding Fe $\gamma \mathrm{R}$ IIIa 158 FF expressing NK cells. The importance of the Fc $\gamma$ R IIIa-158 polymorphism in cytotoxicity of SCCHN cells by NK cells supports a potential role for immune activation and may explain patient variability of cetuximab mediated clinical responses. Cellular and secreted immune profiles and $\mathrm{Fc} \gamma \mathrm{R}$ genotypes from patients' lymphocytes may provide clinically useful biomarkers of immune activation in cetuximab treated patients.

\section{Keywords}

EGFR; Antibodies; ADCC; Immunotherapy

\section{Introduction}

The epidermal growth factor receptor (EGFR) has become one of the most promising therapeutic targets in the treatment of solid malignancies, including squamous cell carcinoma of the head and neck (SCCHN) [1,2]. Immunotherapy with the EGFR-specific IgG1 mAb, cetuximab, significantly improves survival of SCCHN patients with advanced or metastatic disease [3, 4], but responses are limited to $10-20 \%$ of patients. The available evidence is compatible with the possibility that the beneficial effects of cetuximab administration on the clinical course of the disease reflect both inhibition of EGFR tyrosine phosphorylation and triggering of antibody-dependent, cell-mediated cytotoxicity (ADCC) of SCCHN cells. In this lytic reaction, cetuximab coats EGFR, and binds to $\mathrm{Fc} \gamma$ receptors $(\mathrm{Fc} \gamma \mathrm{R})$ expressed on effector cells; this interaction triggers their activation and degranulation, resulting in the lysis of antibody coated target cells. The extent of in vitro ADCC of malignant cells mediated by tumor antigen-specific mAb such as CD20- and HER2-specific mAb is influenced by several variables such as expression of the target antigen and $\mathrm{mAb}$ concentration. More importantly, $\mathrm{Fc} \gamma \mathrm{R}$ IIa and IIIa polymorphisms have been shown to play an important role in the clinical efficacy of mAb-based immunotherapy of malignant disease $[5,6]$. Thus, beneficial clinical responses to immunotherapy with the $\mathrm{CD} 20$-specific $\mathrm{mAb}$ rituximab or with the HER2-specific $\mathrm{mAb}$ trastuzumab are correlated with the Fc $\gamma \mathrm{R}$ IIIa VV polymorphism of the treated patients afflicted with lymphoproliferative diseases and breast carcinoma, respectively. In contrast, a recent report in cetuximab-treated colorectal carcinoma patients suggested that clinical response correlated with the Fc $\gamma \mathrm{R}$ IIIa FF genotype [7].

Despite this discrepancy, the variables which influence the extent of cell-dependent lysis of SCCHN cells mediated by cetuximab have been investigated only to a limited extent, although this information may contribute to our understanding of the mechanism(s) underlying the differential clinical responses of the patients treated with cetuximab-based 
immunotherapy and to optimizing the selection of patients treated with this type of immunotherapy. Therefore, in the present study we have investigated whether the extent of cell-dependent lysis of SCCHN cell lines mediated by cetuximab in vitro is influenced by the Fc $\gamma$ R IIIa-158 genotype expressed by effector NK cells, the level of EGFR expression on target cells, and the cetuximab concentration. Additionally, we have correlated the $\mathrm{Fc} \gamma \mathrm{R}$ IIIa-158 genotype with NK cell activation, degranulation, and cytokine secretion.

\section{Materials and methods}

\section{Cell lines}

The SCCHN cell lines PCI-15B, PCI-30, SCC-4, and UM-22B $[8,9]$ were grown in DMEM supplemented with $10 \% \mathrm{FBS}$ at $37^{\circ} \mathrm{C}$ in a $5 \% \mathrm{CO}_{2}$ atmosphere.

\section{Antibodies}

The EGFR-specific chimeric mAb cetuximab (Erbitux, BMS Imclone, Princeton NJ) was purchased from the University of Pittsburgh Hillman Cancer Center Pharmacy. The CD16specific mAb 3G8 (BD Biosciences, San Jose CA) and a human IgG1 isotype control were purchased from Sigma Aldrich, St. Louis, MO. The CD3-, CD16- CD56-, CD69-, CD16-, and CD107a-specific mAbs were purchased from R \& D Systems (Minneapolis, MN).

\section{Cytokine}

Recombinant human IL-2 and IL-15 were purchased from R \& D Systems.

\section{Cell sorting}

PBMC were obtained from healthy donors through buffy coat lymphocytes purchased from the Western Pennsylvania blood bank. Whole blood or leukapheresis products were centrifuged through a Ficoll-hypaque gradient (Amersham Biosciences, Uppsala, Sweden). Enriched NK cells were obtained from PBMC using the human Easysep NK cell negative selection kit (Stemcell technologies, Vancouver, BC, Canada) according to the manufacturer's protocol. NK cell purity $>95 \%$ was monitored using flow cytometry with CD3-, CD56- and CD16-specific mAbs.

\section{Flow cytometry}

Unfractionated PBMC or purified NK cells ( $\left.>95 \% \mathrm{CD}^{-}, \mathrm{CD}^{+} 6^{+}\right)$at $1 \times 10^{6} / \mathrm{ml}$ in $1 \% \mathrm{BSA} /$ PBS were incubated for $30 \mathrm{~min}$ at room temperature with CD56-specific PE-Cy5.5 labeled mAb, CD69-specific FITC labeled mAb, CD16-specific PE-TR labeled mAb, CD3-specific $\mathrm{PE}$ labeled $\mathrm{mAb}$, and CD107a-specific PE labeled $\mathrm{mAb}$. Following three washings with media, cells were fixed with 2\% PFA and analyzed with a Beckman Coulter EPICS XL flow cytometer. Data were analyzed using the EXPO 32 software (Beckman Coulter, Fullerton, CA).

\section{FcyR Illa genotyping}

The Fc $\gamma \mathrm{R}$ IIIa-158 genotype was determined using a quantitative PCR-based assay kit from Applied Biosystems (Framingham, MA). Briefly, genomic DNA was extracted using the DNeasy Kit (Qiagen, Valencia, CA) following the manufacturer's protocol. Five to $50 \mathrm{ng}$ of genomic DNA were added to a $25-\mu \mathrm{L}$ reaction using $2 \times$ Taqman master mix (Applied Biosystems). Plates were run and analyzed for allelic expression using an ABI prism 7700 sequence detection system. 


\section{Cytotoxicity assays}

Cytotoxicity was determined using a ${ }^{51} \mathrm{Cr}$ or $\mathrm{LDH}$ release assay. Target cells were incubated in $100 \mu \mathrm{L}$ of media with $25 \mu \mathrm{Ci}$ of $\mathrm{Na}_{2}^{51} \mathrm{CrO}_{4}$ (Perkin Elmer, Boston MA) for $60 \mathrm{~min}$ at $37^{\circ} \mathrm{C}$ and resuspended in RPMI 1640 medium supplemented with $25 \mathrm{mM}$ HEPES. Cells were thoroughly washed and plated at various effector: target (E:T) ratios in 96-well plates. Cetuximab was added at the specified concentrations, and peripheral blood mononuclear cells (PBMC) or purified NK cells were added at the specified effector:target (E:T) ratio. Plates were incubated for $4 \mathrm{~h}$ at $37^{\circ} \mathrm{C}$ in a $5 \% \mathrm{CO}_{2}$ atmosphere. Controls for spontaneous (cells only) and maximal lysis (cells treated with $1 \%$ Triton-X) and specificity mAb control (human IgG1 isotype) were included. Each reaction was done in triplicate and repeated three times. The supernatants were collected and analyzed with a Perkin Elmer 96-well plate gamma counter or analyzed for their LDH content using the Roche LDH cytotoxicity assay. Results were normalized with the formula lysis $=$ (experimental lysis - spontaneous lysis $) /$ (experimental lysis - maximal lysis) $\times 100$, NK activity (cytotoxicity with human IgG1 $\mathrm{mAb}$ ) was subtracted, and results were plotted on a graph.

\section{Cytokine measurement}

Cytokine concentrations in the supernatants of cytotoxicity assays were determined using a multiplexed ELISA (Luminex ${ }^{\mathrm{TM}}$ ). Briefly, supernatants collected from the cytotoxicity assays were tested for IFN- $\gamma$, IL-8, MIP $1 a$ MIP $1 \beta$ and TNF- $\gamma$ levels using commercially validated kits (Biosource, Carlsbad, CA). A standard calibration curve was generated for quantification by serial dilutions using recombinant human cytokines as described [10].

\section{Statistical analysis}

Equality of genotype frequencies between SCCHN patients and healthy controls was tested with a chi square test. All reported test results are two tailed. The significance of differences among the three groups was tested using the Kruskal-Wallis test. If the $P$ value was $₫ 0.05$, specific paired contrasts of interest were tested using the Wilcoxon test. All reported test results are two tailed.

\section{Results}

\section{Role of FcyRIII 158 polymorphism in the cetuximab mediated, NK cell dependent in vitro lysis of SCCHN cells}

PBMC lyse UM-22B SCCHN cells incubated with cetuximab [11]. NK cells are the lytic effector cells in PBMC, since their lytic activity in a 4-h ADCC assay is completely abrogated by NK cell depletion (Fig. 1a). The lytic activity of NK cells is mediated by the interaction of $\mathrm{Fc} \gamma \mathrm{R}$ with cetuximab since incubation with the $\mathrm{Fc} \gamma \mathrm{R}$ IIIa-specific mAb 3G8 completely abrogated the activity of NK cells in 4-h ADCC assays (Fig. 1b). The inhibitory activity of mAb 3G8 is specific, since incubation of NK cells with the human IgG1 isotype control $\mathrm{mAb}$ had no detectable effect on the extent of lysis (not shown). The extent of lysis of SCCHN cells is dependent on the E:T ratio and on the cetuximab concentration.

Specifically, NK cell lytic activity was found to increase linearly at higher E:T ratios (from 6:1 to 50:1), at a constant cetuximab concentration $(1 \mu \mathrm{g} / \mathrm{ml})$. Cetuximab-induced ADCC against SCCHN cells reached a plateau above approximately $1 \mu \mathrm{g} / \mathrm{ml}$, regardless of the Fc $\gamma$ R IIIa genotype expressed by the NK cells (Fig. 1c). Therefore, all further experiments were performed with cetuximab concentrations $>1 \mu \mathrm{g} / \mathrm{ml}$.

To determine whether polymorphism of the Fc $\gamma \mathrm{R}$ IIIa receptor (Table 1), which is primarily expressed by NK cells, plays a role in the extent of NK-cell dependent, cetuximab-mediated lysis of SCCHN cells, NK cells expressing the Fc $\gamma$ R IIIa-158 VV, VF or FF genotypes were 
compared in their ability to lyse SCCHN cells coated with cetuximab in a 4-h ADCC assay. Our analysis of cetuximab-mediated ADCC focused on the codon 158 polymorphism of the $\mathrm{Fc} \gamma \mathrm{R}$ IIIa receptor. The mean lytic activity of NK cells expressing the Fc $\gamma \mathrm{R}$ IIIa-158 VV or VF was significantly higher than that of NK cells expressing the Fc $\gamma$ R-IIIa FF genotype, irrespective of the cetuximab concentration used. As shown in Fig. 1b, the presence of a $\mathrm{V}$ allele correlated with the highest cytotoxicity $(P<0.001)$.

\section{Association of FcyRIlla-158 genotype with the extent of NK cell activation phenotype and the level of cytokine secretion}

Since the level of cetuximab-mediated lysis of SCCHN cells by NK cells correlated significantly with their Fc $\gamma \mathrm{R}$ IIIa-158 genotype, additional experiments investigated its association with the extent of NK cell activation following incubation with cetuximab coated SCCHN cells. To this end, purified NK cells, expressing Fc $\gamma$ R IIIa-158 VV or FF, were incubated for $4 \mathrm{~h}$ at room temperature with UM-22B SCCHN cells in the presence cetuximab $(1 \mu \mathrm{g} / \mathrm{ml})$. Then NK cells were stained with CD56-, CD69- and CD107a-specific $\mathrm{mAb}$ and analyzed by multicolor flow cytometry. This analysis showed that the activation markers CD 69 and CD 107a were expressed by a significantly $(P<0.05)$ higher percentage of NK cells with the Fc $\gamma$ RIIIa-158 VV genotype than of NK cells with the Fc $\gamma$ R IIIa-158 FF genotype, a finding consistent with the extent of lytic activity displayed by NK cells with different genotypes. Representative plots are shown in Fig. 2a.

To investigate whether cytokine secretion was associated with lytic degranulation, the supernatants from these ADCC cultures were analyzed for the content of cytokines and chemokines associated with NK cell activation, and T cell chemoattraction using a multiplexed ELISA (Luminex ${ }^{\mathrm{TM}}$ ). In agreement with the results derived from the analysis of the activation phenotype of NK cells expressing different Fc $\gamma$ RIIIa genotype, the Fc $\gamma$ RIIIa-158 VV expressing NK cells secreted significantly higher levels of IFN- $\gamma$, IL-8, MIP- $1 a$, MIP- $1 \beta$, RANTES, and TNF- $a$ than NK cells expressing the Fe $\gamma$ RIIIa- 158 FF genotype ( $P<0.05$, Fig. 2 b). These results were reproduced independently utilizing NK cells from at least three donors of each genotype. On the other hand, no differences were detected in the levels of IFN- $a$, IL-2 or IL-15 in the supernatants harvested from ADCC assays using NK cells expressing different $\mathrm{Fc} \gamma$ RIIIa-158 genotypes (data not shown).

\section{Enhancement by cytokines of the lytic activity and activation marker expression level by NK cells with the poor responder FcyR Illa FF genotype}

To determine whether the lytic activity and the expression level of activation markers by NK cells with the Fc $\gamma$ RIIIa-158 FF genotype could be enhanced by incubation with cytokines, FF-expressing NK cells from six healthy donors were incubated with IL-2 $(20 \mathrm{IU} / \mathrm{ml})$ or IL-15 $(10 \mathrm{ng} / \mathrm{ml})$ for $24 \mathrm{~h}$ at $37^{\circ} \mathrm{C}$. These cells were then tested for their lytic activity in a 4$\mathrm{h}$ ADCC assay which utilized SCCHN cells as targets and cetuximab $(1 \mu \mathrm{g} / \mathrm{ml})$. NK cells displayed significantly (Kruskal-Wallis test $P<0.05$ ) higher lytic activity after incubation with IL-2 or IL-15 (Fig. 3a). Furthermore, the expression level of the activation markers CD69 and CD107a by IL-2 or IL-15 treated NK cells with the Fc $\gamma$ RIIIa-158 FF genotype approached that of NK cells with the Fc $\gamma$ R IIIa-158 VV (Fig. 2a). Lastly, using a multiplexed ELISA, supernatants harvested from the ADCC assays showed a significant increase in the levels of secreted IFN- $\gamma$, IL-8, MIP- $1 a$, MIP-1 $\beta$, and TNF- $a,(P<0.05$, two tailed, Fig. 3b).

\section{Differential effect of the level of EGFR expression by SCCHN cells on their susceptibility to cetuximab-mediated lysis by NK cells expressing different FcyRIlla genotypes}

To investigate the effect of the level of EGFR expression by SCCHN cells on their susceptibility to cetuximab-mediated lysis by NK cells with different $\mathrm{Fc} \gamma \mathrm{R}$ IIIa genotypes, 
the SCCHN cell lines PCI-15B, PCI-30 and SCC-4 which express different EGFR levels were tested for their susceptibility to cetuximab-mediated lysis by NK cells expressing the $\mathrm{Fc} \gamma \mathrm{R}$ IIIaFF, the Fc $\gamma \mathrm{R}$ IIIaVF, or the Fc $\gamma \mathrm{R}$ IIIaVV genotypes. As shown in Fig. $4 \mathrm{a}$, an association was found between the EGFR expression level on SCCHN cell lines and the extent of lysis when EGFR level was high; this association became less marked with a decrease in EGFR expression. It is noteworthy that even when the EGFR expression level was high, cetuximab-mediated lysis of SCCHN cell lines by NK cells expressing the Fc $\gamma \mathrm{R}$ IIIa FF allele was barely detectable.

Since the SCCHN cells lines used as targets are allogeneic, the potential effect of variables other than EGFR expression on the extent of target cell lysis cannot be excluded. To overcome this limitation, we investigated the role of EGFR level in the extent of cetuximabmediated lysis by NK cells, utilizing as targets derivatives of a SCCHN cell line in which the EGFR expression level was varied by RNA interference (RNAi). To this end, the EGFR $^{+}$SCCHN cell lines PCI-15B, PCI-30, SCC-4, and UM-22B were incubated with EGFR specific RNAi oligonucleotides for $48 \mathrm{~h}$ at $37^{\circ} \mathrm{C}$. EGFR expression was consistently knocked down $75-80 \%$ as determined by cetuximab staining and flow cytometric analysis (not shown). The EGFR knockdown was specific, since no change was detected in EGFR expression on SCC-4 cells incubated with scrambled control RNAi oligonucleotides under the same experimental conditions.

Parental PCI-15B, PCI-30, SCC-4 cells and UM-22B, and PCI-15B, PCI-30, SCC-4, and UM-22B cells after EGFR knockdown, respectively, were compared in their susceptibility to lysis in 4-h ADCC assays (Fig. 4b). In these assays, cetuximab was used at concentration of $10 \mu \mathrm{g} / \mathrm{ml}$, and purified NK cells expressing the Fc $\gamma \mathrm{R}$ IIIa-158 VF genotype were used as effectors. In three of the four SCCHN cell lines, under the experimental conditions used, knockdown of EGFR was associated with a reduction in the extent of cetuximab-mediated lysis of SCCHN cells.

\section{Lack of detectable effect of EGFR vIll variant on the susceptibility of SCCHN cell lines to cetuximab-mediated, NK-cell-dependent lysis}

Mutant forms of EGFR have been linked to several tumor types, with the most common mutant in SCCHN being the variant III (vIII) EGFR mutation. This extracellular domain truncation mutant which possesses ligand-independent receptor tyrosine kinase activity is found in approximately $42 \%$ of SCCHN tumors and has been reported to bind cetuximab weakly [13]. To determine whether the vIII mutant has an effect on cetuximab-mediated lysis of SCCHN cells by NK cells, UM-22B cells stably transfected with the vIII EGFR mutant were compared to the parental cells in their susceptibility to lysis in 4-h ADCC assays. Purified NK cells expressing Fc $\gamma$ R IIIa-158 VF from five donors were used as effectors at an E:T ratio of 20:1; cetuximab was used at concentrations ranging from $0.1 \mathrm{ng} /$ $\mathrm{ml}$ to $50 \mu \mathrm{g} / \mathrm{ml}$. Cells transfected with a control vector were used as a control. Figure 5 shows that the presence of the vIII EGFR variant had no detectable effect on ADCC at any of the cetuximab concentrations tested.

\section{Discussion}

Despite the clinical efficacy seen in the phase III trials of cetuximab as therapy for SCCHN and colorectal cancer, the exact mechanism(s) of action of this mAb is not certain, and responses are seen only in a subset of 10-20\% of treated patients. Importantly, cetuximab treatment of SCCHN cells in vitro does not induce tumor cell apoptosis or lysis, and this only occurs when lymphocytes are added to the culture, supporting an immune-mediated mechanism contributing to cetuximab's antitumor effect. EGFR signaling blockade is likely to play a role in the clinical responses seen in patients receiving cetuximab $[12,14]$. 
However, the immune complexes formed on the surface of tumor cells treated with this agent provide an additional mechanism of tumor cell lysis [11], as demonstrated in vivo in other tumor types $[15,16]$ and as we have demonstrated in vitro. Additional evidence in support of the role of immune-mediated responses in cetuximab therapy is provided by the lack of correlation between level of EGFR expression and clinical responses to cetuximab therapy, and by the recently reported correlation between certain $\mathrm{Fc} \gamma \mathrm{R}$ genotypes and clinical responses to single-agent cetuximab in colorectal cancer patients [7]. However, there is a conflict in the literature as to the exact $\mathrm{Fc} \gamma \mathrm{R}$ IIIa- 158 genotype which mediates optimal lytic activity and is correlated with a better clinical outcome. Thus a better understanding of a mAb-mediated immune mechanism is crucial, since lack of this information presents a major barrier to improving clinical responses and circumventing cetuximab resistance in vivo.

In the present study, we extended our previous observations that cetuximab mediates celldependent cytotoxicity against SCCHN cells [11]. We found that the expression of an $\mathrm{Fc} \gamma \mathrm{R}$ IIIa-158 V allele correlates strongly with ADCC activity, NK cell activation, degranulation and secretion of several cytokines with anti-tumor activity. These findings provide in vitro evidence that cetuximab can mediate biologically important immune cell activation and tumor cell lysis, both of which may occur in patients receiving this mAb [17], as observed with trastuzumab treated patients.

As mentioned earlier, our in vitro results are in agreement with those obtained for ADCC mediated by other mAbs, such as rituximab or trastuzumab, where the $\mathrm{Fc} \gamma \mathrm{R}$ IIIa VV allele demonstrates stronger antitumor activity. However, our data conflict with those of Zhang et al. [7] who found a correlation between the $\mathrm{Fc} \gamma \mathrm{R}$ III a FF and clinical response to single agent cetuximab in metastatic colorectal cancer. The latter report contradicts extensive in vitro data showing that the $\mathrm{Fc} \gamma \mathrm{R}$ IIIa-158 VV allele has a better binding to IgG1 antibodies $[18,19]$ and is correlated with a favorable clinical outcome in patients receiving rituximab as a treatment of follicular lymphoma [16]. In contrast, the FF polymorphism was correlated with lower response rates and decreased survival. Several reasons may be considered for this discrepancy. First, the trial by Zhang et al. [7] enrolled only 39 heavily pretreated patients, and found a marginal correlation with this polymorphism. Also, a second $\mathrm{Fc} \gamma \mathrm{R}$ polymorphism, Fc $\gamma$ R IIa-131 was correlated with clinical response in this study, complicating the interpretation and possible comparisons with more controlled in vitro studies, as described by others $[15,16]$ and in the present study. Furthermore, $\mathrm{Fc} \gamma \mathrm{R}$ polymorphisms have not been correlated with responses to rituximab therapy in diffuse large $\mathrm{B}$ cell lymphomas, another group of $\mathrm{CD} 20^{+}$malignancies [20], suggesting that immune activation in $\mathrm{mAb}$-based therapies might be tumor type specific. Thus, one must also consider that different tumor cell populations may explain the variability of these results. Finally, type I error exists as a potential explanation for the different results obtained by Zhang et al. [7] and by ourselves. The increase in risk of progression-free survival (PFS) associated with FF is 2.28 (95\% CI $=0.78-6.63)$, and the adjusted $P$ value $=0.114$. The conclusions derived from this study which used cetuximab as a single-agent, a rarely used regimen, may not be applicable to the widely used regimens, which combine cetuximab with chemo-therapy or radiotherapy.

Our study shows conclusively that EGFR level of expression has an impact on cetuximabmediated ADCC, and we have shown this effect by modulating EGFR expression on syngeneic cell lines. This finding is consistent with the correlation between EGFR expression levels and the extent of cell-dependent lysis mediated by cetuximab which has been observed in several in vitro studies [24, 25], including our own. It is noteworthy that our study has avoided the potential interference of variables other than EGFR expression level which may affect the susceptibility of different cell lines to cell-dependent lysis, since 
autologous cell lines with different EGFR expression levels were used as targets in ADCC. In our experiment, lysis of PCI30 had a blunted response to alterations in EGFR levels. This may be due to some intrinsic variable(s) that make(s) PCI30 susceptible to ADCC even at low levels of antibody binding. Our in vitro results conflict with studies which have shown that clinical responses to cetuximab-mediated therapy are not correlated with EGFR expression levels [21-23]. Level of EGFR expression in vitro influences the extent of lysis at all doses of cetuximab, but its effect is more pronounced in the presence of NK cells expressing the favorable $\mathrm{Fc} \gamma \mathrm{R}$ genotype (VV), and no study to date has correlated clinical outcome with both Fc $\gamma \mathrm{R}$ IIIa genotype and EGFR level of expression as combined markers.

The EGFR vIII truncation mutant is found in $>40 \%$ of SCCHN tumors and affords these tumors with a growth advantage as well as potential for resistance to cetuximab treatment [13]. Because cetuximab is a competitive antagonist of EGF and the vIII mutant's downstream signaling has been uncoupled from EGF binding, mechanistically it is consistent that this mutant would confer resistance to that pathway of treatment. However, it has been shown that cetuximab binds this mutant form of EGFR [26-28], and these data leave open the possibility of an immune-modulated response to cetuximab binding to the mutant receptor. Alternatively, the vIII mutant might interfere with cetuximab binding to wild type EGFR. Our study demonstrates that the vIII mutant causes no detectable resistance to cetuximab-mediated, cell-dependent cytotoxicity of SCCHN cells when compared to wild type EGFR expressing cells. We note that the strength of these data is enhanced by the concurrent expression of wild-type EGFR in the stably transfected UM-22B cell line, which is also the case in vivo as all tumors that possess the vIII mutant form also express the wildtype form of EGFR.

Several implications for cetuximab based clinical cancer therapy are apparent based on our results. First, the selection of the most appropriate candidates for cetuximab treatment could be based on specific $\mathrm{Fc} \gamma \mathrm{R}$ IIIa-158 geno-types, a parameter that could be used as a selection criterion or predictive biomarker of clinical responses. Second, monitoring serum concentrations of specific cytokines such as IFN- $\gamma$, TNF- $a$, MIP- $1 a$, MIP- $1 \beta$, or a combination of these would facilitate real-time immune correlates of treatment efficacy and may serve as an early predictor of responses prior to clinical response (i.e. tumor shrinkage, RECIST criteria or treatment resistance). In this regard, we have previously used multiplex cytokine profiles to distinguish between patients with or without evidence of disease [29], an analysis that would be feasible to perform related to treatment efficacy. We should keep in mind that other variables have an impact on cetuximab mediated ADCC. Among these are the triggering of tumor antigen-specific $\mathrm{T}$ cells and potential interference of cell-mediated lysis by immune escape mechanisms [30].

\section{Acknowledgments}

This work has been funded by a pilot grant from the American Head and Neck Society (ALA and RLF) and R01 DE19727 (RLF).

\section{References}

1. Ang KK, Berkey BA, Tu X, et al. Impact of epidermal growth factor receptor expression on survival and pattern of relapse in patients with advanced head and neck carcinoma. Cancer Res. 2002; 62(24):7350-7356. [PubMed: 12499279]

2. Grandis JR, Tweardy DJ. Elevated levels of transforming growth factor alpha and epidermal growth factor receptor messenger RNA are early markers of carcinogenesis in head and neck cancer. Cancer Res. 1993; 53(15):3579-3584. [PubMed: 8339264]

3. Bonner JA, Harari PM, Giralt J, et al. Radiotherapy plus cetuximab for squamous-cell carcinoma of the head and neck. N Engl J Med. 2006; 354(6):567-578. [PubMed: 16467544] 
4. Vermorken, JB.; Mesia, R.; Vega-Villegas, ME., et al. Cetuximab in combination with cisplatin or carboplatin and 5-fluorouracil (5-FU) in the first-line treatment of patients with recurrent and/or metastatic squamous cell carcinoma of the head and neck (R\&M SCCHN) (EXTREME).. ASCO annual meeting proceedings; 2006.

5. Taylor C, Hershman D, Shah N, et al. Augmented HER-2 specific immunity during treatment with trastuzumab and chemotherapy. Clin Cancer Res. 2007; 13(17):5133-5143. [PubMed: 17785568]

6. Varchetta S, Gibelli N, Oliviero B, et al. Elements related to heterogeneity of antibody-dependent cell cytotoxicity in patients under trastuzumab therapy for primary operable breast cancer overexpressing Her2. Cancer Res. 2007; 67(24):11991-11999. [PubMed: 18089830]

7. Zhang W, Gordon M, Schultheis AM, et al. FCGR2A and FCGR3A polymorphisms associated with clinical outcome of epidermal growth factor receptor expressing metastatic colorectal cancer patients treated with single-agent cetuximab. J Clin Oncol. 2007; 25(24):3712-3718. [PubMed: 17704420]

8. Lin CJ, Grandis JR, Carey TE, et al. Head and neck squamous cell carcinoma cell lines: established models and rationale for selection. Head Neck. 2007; 29(2):163-188. [PubMed: 17312569]

9. Thomas SM, Zeng Q, Epperly MW, et al. Abrogation of head and neck squamous cell carcinoma growth by epidermal growth factor receptor ligand fused to pseudomonas exotoxin transforming growth factor alpha-PE38. Clin Cancer Res. 2004; 10(20):7079-7087. [PubMed: 15501988]

10. Hathaway B, Landsittel DP, Gooding W, et al. Multiplexed analysis of serum cytokines as biomarkers in squamous cell carcinoma of the head and neck patients. Laryngoscope. 2005; 115(3):522-527. [PubMed: 15744170]

11. Lopez-Albaitero A, Ferris RL. Immune activation by epidermal growth factor receptor specific monoclonal antibody therapy for head and neck cancer. Arch Otolaryngol Head Neck Surg. 2007; 133(12):1277-1281. [PubMed: 18086972]

12. Robert F, Ezekiel MP, Spencer SA, et al. Phase I study of anti-epidermal growth factor receptor antibody cetuximab in combination with radiation therapy in patients with advanced head and neck cancer. J Clin Oncol. 2001; 19(13):3234-3243. [PubMed: 11432891]

13. Sok JC, Coppelli FM, Thomas SM, et al. Mutant epidermal growth factor receptor (EGFRvIII) contributes to head and neck cancer growth and resistance to EGFR targeting. Clin Cancer Res. 2006; 12(17):5064-5073. [PubMed: 16951222]

14. Kalyankrishna S, Grandis JR. Epidermal growth factor receptor biology in head and neck cancer. J Clin Oncol. 2006; 24(17):2666-2672. [PubMed: 16763281]

15. Musolino A, Naldi N, Bortesi B, et al. Immunoglobulin $G$ fragment $C$ receptor polymorphisms and clinical efficacy of trastuzumab-based therapy in patients with HER-2/neu-positive metastatic breast cancer. J Clin Oncol. 2008; 26(11):1789-1796. [PubMed: 18347005]

16. Weng WK, Levy R. Two immunoglobulin G fragment C receptor polymorphisms independently predict response to rituximab in patients with follicular lymphoma. J Clin Oncol. 2003; 21(21): 3940-3947. [PubMed: 12975461]

17. Roda JM, Joshi T, Butchar JP, et al. The activation of natural killer cell effector functions by cetuximab-coated, epidermal growth factor receptor positive tumor cells is enhanced by cytokines. Clin Cancer Res. 2007; 13(21):6419-6428. [PubMed: 17962339]

18. Dall'Ozzo S, Tartas S, Paintaud G, et al. Rituximab-dependent cytotoxicity by natural killer cells: influence of FCGR3A polymorphism on the concentration-effect relationship. Cancer Res. 2004; 64(13):4664-4669. [PubMed: 15231679]

19. Cartron G, Dacheux L, Salles G, et al. Therapeutic activity of humanized anti-CD20 monoclonal antibody and polymorphism in IgG Fc receptor FcgammaRIIIa gene. Blood. 2002; 99(3):754-758. [PubMed: 11806974]

20. Mitrovic Z, Aurer I, Radman I, Ajdukovic R, Sertic J, Labar B. FCgammaRIIIA and FCgammaRIIA polymorphisms are not associated with response to rituximab and CHOP in patients with diffuse large B cell lymphoma. Haematologica. 2007; 92(7):998-999. [PubMed: 17606457]

21. Lu Y, Li X, Liang K, et al. Epidermal growth factor receptor (EGFR) ubiquitination as a mechanism of acquired resistance escaping treatment by the anti-EGFR monoclonal antibody cetuximab. Cancer Res. 2007; 67(17):8240-8247. [PubMed: 17804738] 
22. Wild R, Fager K, Flefleh C, et al. Cetuximab preclinical antitumor activity (monotherapy and combination based) is not predicted by relative total or activated epidermal growth factor receptor tumor expression levels. Mol Cancer Ther. 2006; 5(1):104-113. [PubMed: 16432168]

23. Psyrri A, Egleston B, Weinberger $\mathrm{P}$, et al. Correlates and determinants of nuclear epidermal growth factor receptor content in an oropharyngeal cancer tissue microarray. Cancer Epidemiol Biomarkers Prev. 2008; 17(6):1486-1492. [PubMed: 18559565]

24. Kawaguchi Y, Kono K, Mimura K, Sugai H, Akaike H, Fujii H. Cetuximab induce antibodydependent cellular cytotoxicity against EGFR-expressing esophageal squamous cell carcinoma. Int J Cancer. 2007; 120(4):781-787. [PubMed: 17096332]

25. Kurai J, Chikumi H, Hashimoto K, et al. Antibody-dependent cellular cytotoxicity mediated by cetuximab against lung cancer cell lines. Clin Cancer Res. 2007; 13(5):1552-1561. [PubMed: 17332301]

26. Aerts HJ, Dubois L, Hackeng TM, et al. Development and evaluation of a cetuximab-based imaging probe to target EGFR and EGFRvIII. Radiother Oncol. 2007; 83(3):326-332. [PubMed: 17531336]

27. Yang W, Wu G, Barth RF, et al. Molecular targeting and treatment of composite EGFR and EGFRvIII-positive gliomas using boronated monoclonal antibodies. Clin Cancer Res. 2008; 14(3): 883-891. [PubMed: 18245552]

28. Patel D, Lahiji A, Patel S, et al. Monoclonal antibody cetuximab binds to and down-regulates constitutively activated epidermal growth factor receptor vIII on the cell surface. Anti-cancer Res. 2007; 27(5A):3355-3366.

29. Linkov F, Lisovich A, Yurkovetsky Z, et al. Early detection of head and neck cancer: development of a novel screening tool using multiplexed immunobead-based biomarker profiling. Cancer Epidemiol Biomarkers Prev. 2007; 16(1):102-107. [PubMed: 17220337]

30. Kuss I, Hathaway B, Ferris RL, Gooding W, Whiteside TL. Decreased absolute counts of T lymphocyte subsets and their relation to disease in squamous cell carcinoma of the head and neck. Clin Cancer Res. 2004; 10(11):3755-3762. [PubMed: 15173082] 

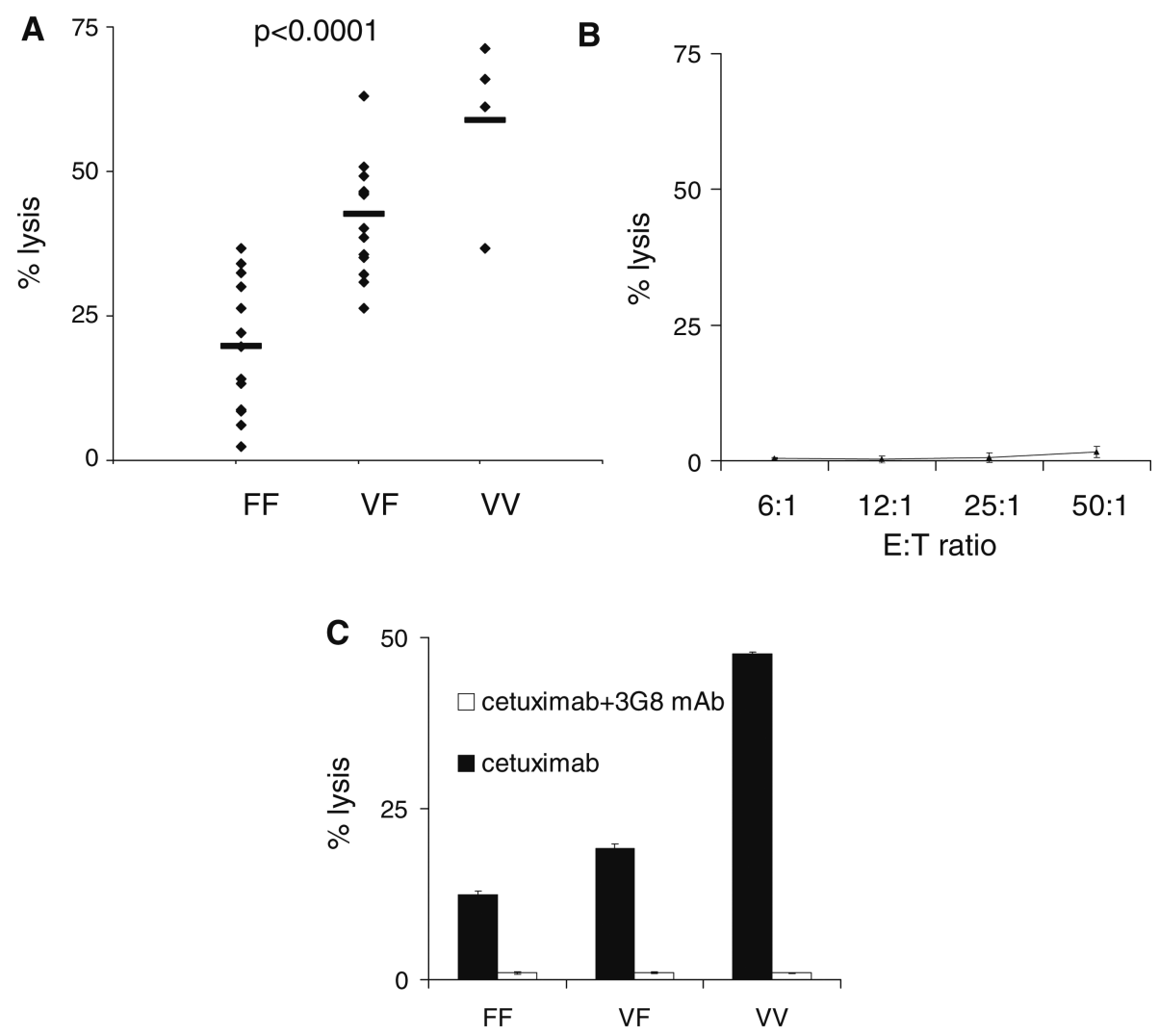

Fig. 1.

Association of the extent of lysis in ADCC by healthy donor PBMC with Fc $\gamma \mathrm{R}$ polymorphism. a PBMC (solid curve) or NK-depleted PBMC (dotted curve) were used as effector cells against cetuximab $(1 \mu \mathrm{g} / \mathrm{ml})$ - treated UM-22B cells in a $4 \mathrm{~h}{ }^{51} \mathrm{Cr}$ release cytotoxicity assay at various E:T ratios. b NK cells from donors expressing each Fc $\gamma$ RIIIa codon 158 polymorphism were incubated with or without $10 \mu \mathrm{g} / \mathrm{ml}$ of the FcRIIIa-specific $\mathrm{mAb} 3 \mathrm{G} 8$ before use as effectors in a $4-\mathrm{h}{ }^{51} \mathrm{Cr}$ release ADCC assay against cetuximab treated $(1 \mu \mathrm{g} / \mathrm{ml})$ UM-22B cells. The differences in the extent of lysis in ADCC by NK cells with different genotypes were highly significant $(P<0.0001$ Kruskal-Wallis test). c Effect of cetuximab dose on in vitro ADCC using NK cells expressing Fc $\gamma \mathrm{R}$ IIIa VV, VF or FF genotypes 

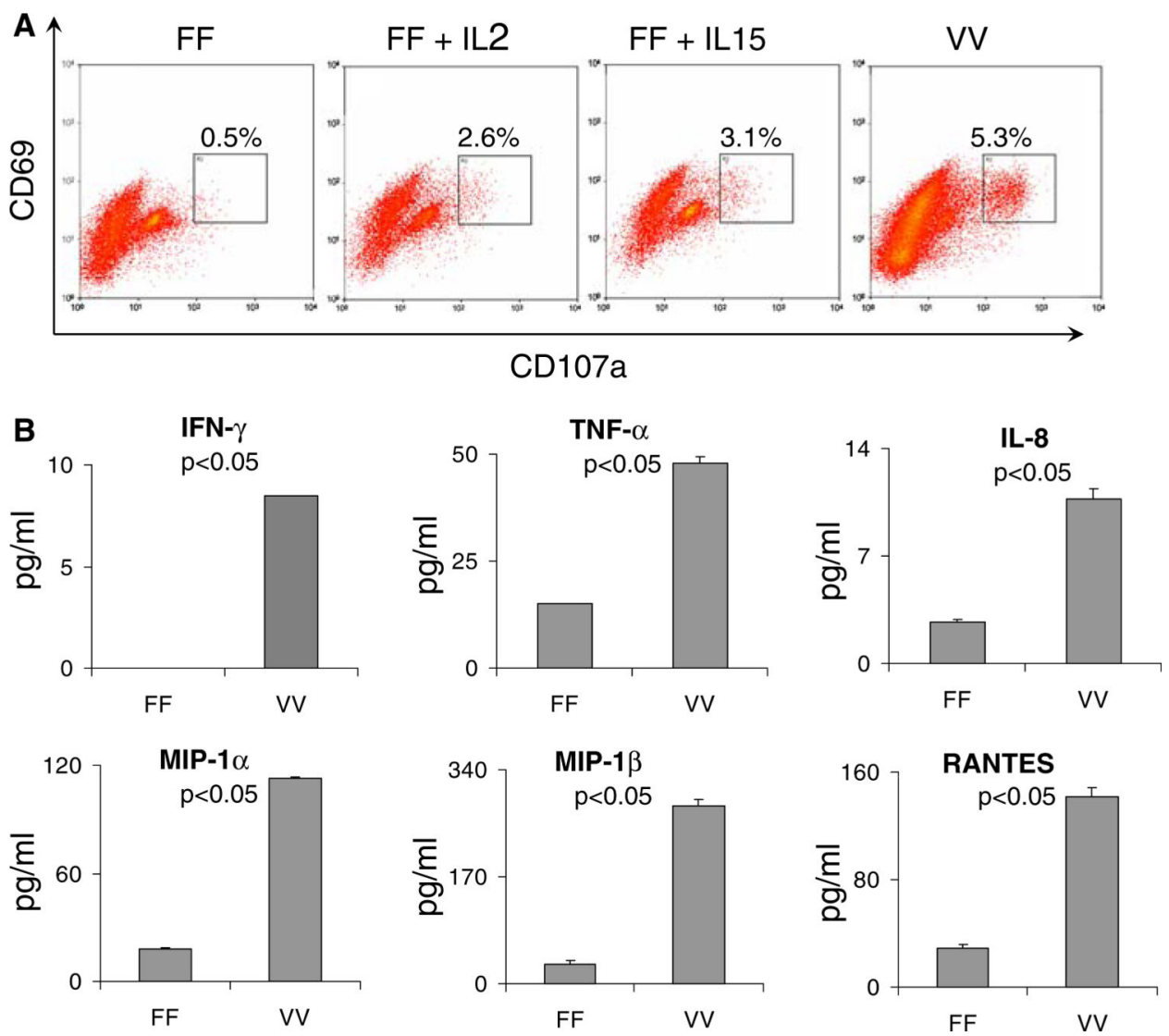

Fig. 2.

NK cell activation and cytokine secretion correlate with level of ADCC mediated by respective $\mathrm{Fc} \gamma \mathrm{R}$ genotype. Healthy donor NK cells together expressing $\mathrm{Fc} \gamma \mathrm{R}$ IIIa-158 VV or FF alleles ( $n=4$ donors per genotype) were used in $4 \mathrm{~h}$ ADCC assays against cetuximab $(1 \mu \mathrm{g} / \mathrm{ml})$ treated UM-22B SCCHN cells. a Effector cells from this assay, pretreated for 18 $\mathrm{h}$ with IL-2 (20 IU/ml) or IL-15 (10 ng/ml), or media alone, were stained for CD69 and CD107a expression by flow cytometry. Results shown are based on an electronically gated $\mathrm{CD} 16^{+} \mathrm{CD}^{2} 6^{+}$population and are representative of three separate experiments. b Supernatants of each ADCC assay were analyzed for their levels of cytokines using a multiplexed ELISA (Luminex ${ }^{\mathrm{TM}}$ technology) as described in materials and methods 
$\mathbf{A}$
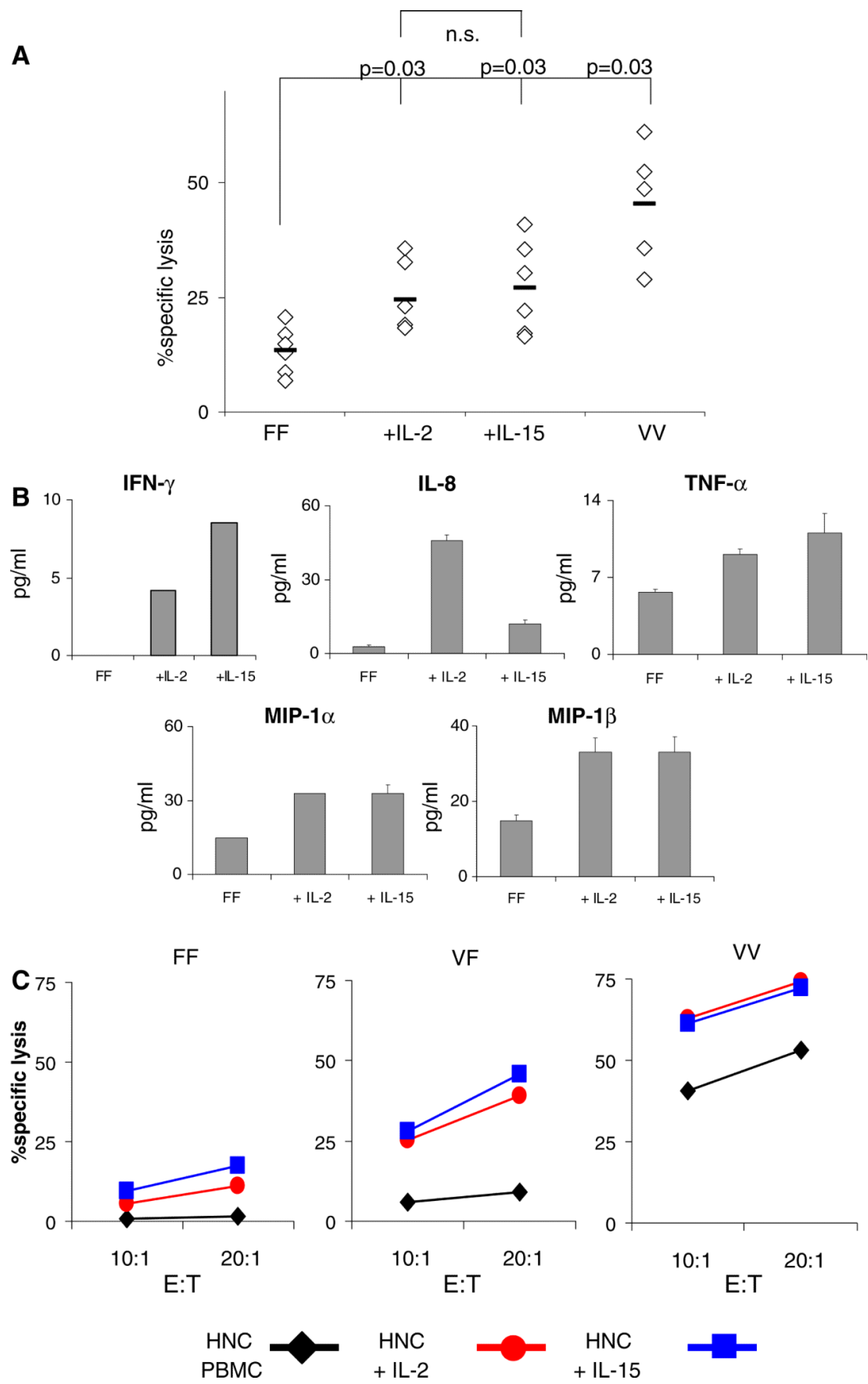

Fig. 3.

Cytokine treatment restores ADCC activity in poor responding Fc $\gamma \mathrm{R}$ IIIa FF genotype. a Purified healthy donor NK cells expressing either the poor binding Fc $\gamma$ RIIIa-158 FF genotype or the high affinity VV genotype were treated for $18 \mathrm{~h}$ with IL-2 (20 IU/ml) or IL-15 $(10 \mathrm{ng} / \mathrm{ml})$, or media alone. NK cells were washed before incubation in a cytotoxicity assay with cetuximab $(1 \mu \mathrm{g} / \mathrm{ml})$ coated, ${ }^{51} \mathrm{Cr}$ labeled UM-22B target cells for $4 \mathrm{~h}$ at an E:T ratio of 20:1. b Supernatants of the ADCC assays in Fig. 3a except the VV were harvested and analyzed using multiplexed ELISA (Luminex ${ }^{\mathrm{TM}}$ ) to quantitate levels of cytokines and chemokines shown. Data are the average of six experiments 
A
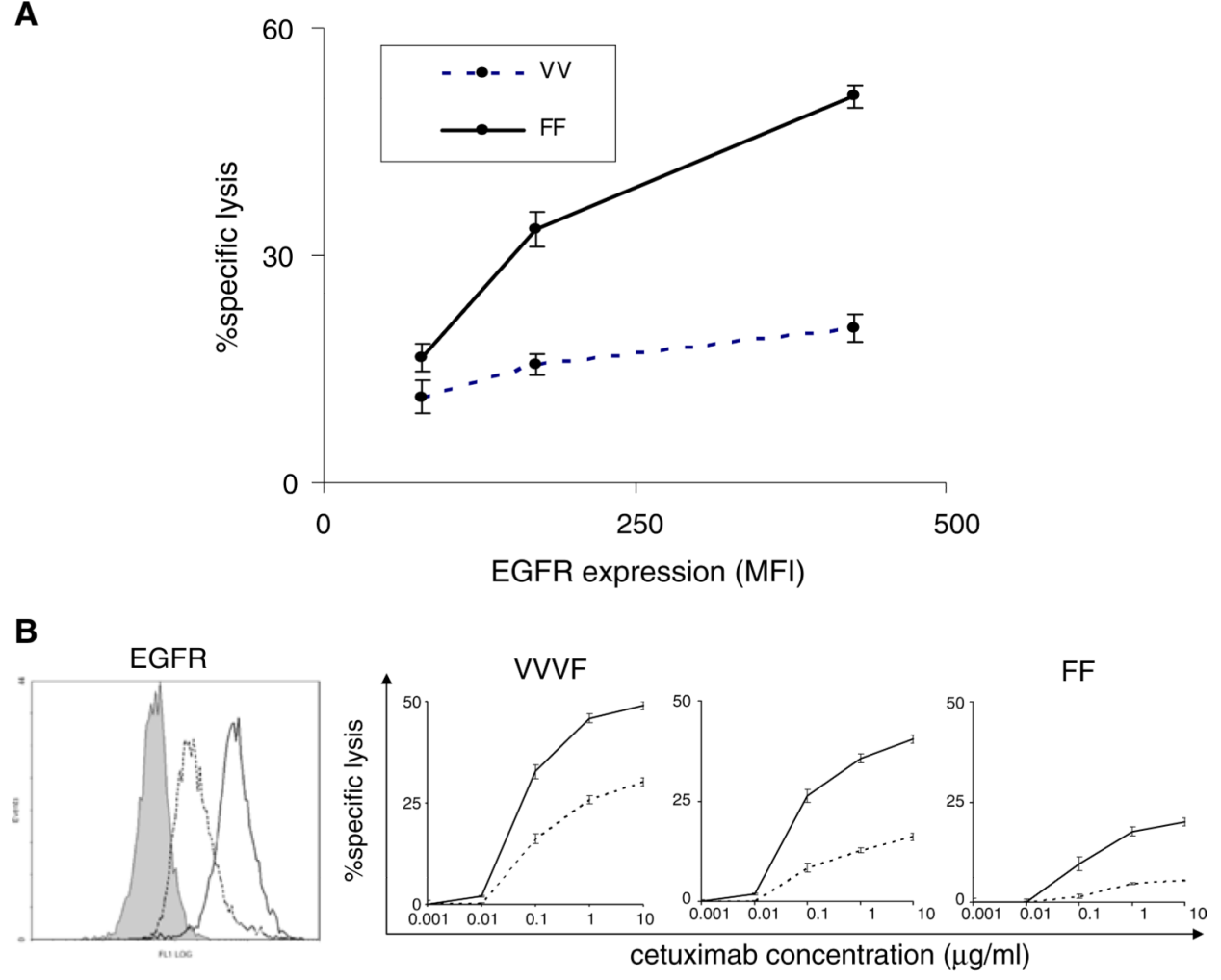

C
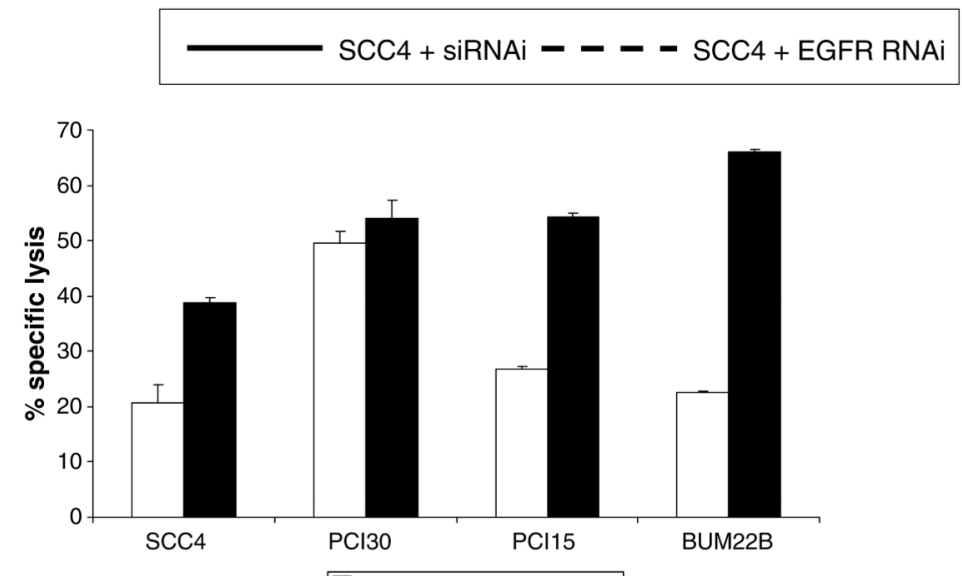

Fig. 4.

Effect of EGFR expression level on cetuximab mediated ADCC. a The SCCHN cell lines SCC4, PCI-15B, and PCI-30 with various EGFR expression levels were used as targets in a 4-h ADCC assay; purified NK cells expressing either Fc $\gamma \mathrm{R} \mathrm{IIIa} \mathrm{VV} \mathrm{or} \mathrm{FF} \mathrm{alleles} \mathrm{were} \mathrm{used}$ at an E:T ratio of 20:1. SCCHN cell targets were incubated with cetuximab at $1 \mu \mathrm{g} / \mathrm{ml}$ for 30 min at $37^{\circ} \mathrm{C}$ before incubation with NK cells. b UM-22B, SCC4, PCI-15B, and PCI-30 cells were transfected with 100nM of EGFR-specific or scrambled control RNAi oligonucleotides for $48 \mathrm{~h}$ before use in a 4-h cytotoxicity assay; purified $\mathrm{Fc} \gamma \mathrm{R}$ IIIa VF-expressing NK cells were used at an E:T ratio of 20:1. Data are the average of four experiments 


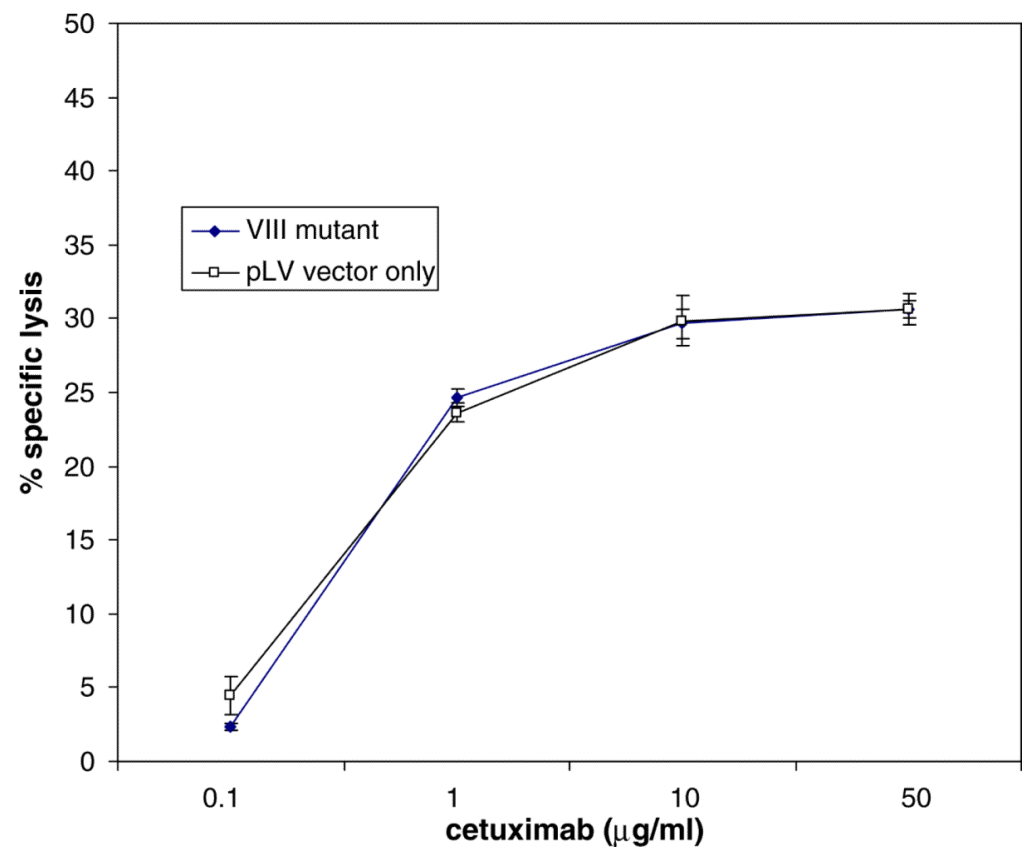

Fig. 5.

Effect of EGFR vIII variant on cetuximab mediated ADCC. UM-22B cells stably transfected with EGFR vIII variant or control vector were used as targets in a 4-h cytotoxicity assay at an E:T ratio of 20:1. The range of cetuximab concentrations used is shown. NK cells expressing Fc $\gamma \mathrm{R}$ IIII VF from five separate donors were tested. Data shown are the average of four experiments 
Table 1

Prevalence of Fc rrIIIa genotypes in HNC patients and healthy controls

\begin{tabular}{lcl}
\hline Genotype & HNC patients $\boldsymbol{N}(\boldsymbol{\%})$ & Healthy controls $\boldsymbol{N}(\boldsymbol{\%})$ \\
\hline VV & $12(7.0)$ & $16(13.6)$ \\
VF & $80(46.5)$ & $65(55.1)$ \\
FF & $80(46.5)$ & $37(31.3)$ \\
Total & $172(100)$ & $37(100)$ \\
\hline
\end{tabular}

